\title{
Conjectural Variations in Aggregative Games: An Evolutionary Perspective
}

\author{
Alex Possajennikov* \\ University of Nottingham
}

Final version accepted for Mathematical Social Sciences, July 2015

Published version available at http://dx.dox.org/10.1016/j.mathsocsci.2015.07.003

\begin{abstract}
Suppose that in symmetric aggregative games, in which payoffs depend only on a player's strategy and on an aggregate of all players' strategies, players have conjectures about the reaction of the aggregate to marginal changes in their strategy. The players play a conjectural variation equilibrium, which determines their fitness payoffs. The paper shows that only consistent conjectures can be evolutionarily stable in an infinite population, where a conjecture is consistent if it is equal to the marginal change in the aggregate determined by the actual best responses. In the finite population case, only zero conjectures representing aggregate-taking behavior can be evolutionarily stable.

Keywords: conjectural variations, aggregative games, indirect evolution, evolutionary stability
\end{abstract}

JEL Codes: C72, D84

${ }^{*}$ School of Economics, University of Nottingham, University Park, Nottingham NG7 2RD, United Kingdom. Tel: +44 115 9515461, fax: +44 115 9514159, email: alex.possajennikov@nottingham.ac.uk, http://www.nottingham.ac.uk/ lezap. 


\section{Introduction}

This paper shows that in symmetric aggregative games there is a link between certain properties of conjectures and the evolutionary success of those conjectures. An aggregative game is a game in which the payoff of a player depends only on the player's own strategy and on an aggregate of the strategies of all players. A typical example of an aggregative game is the Cournot oligopoly; aggregative games were analyzed e.g. in Corchón (1994) and more recently in Cornes and Hartley (2012) and Acemoglu and Jensen (2013), where further examples of such games are discussed.

Conjectures, also called conjectural variations, describe players' beliefs about the reaction of other players to a change in a player's strategy. Theoretically, they allow for an extended range of behaviors (see Figuières et al., 2004, for a book-length discussion of the theory of conjectures and for further references). ${ }^{1}$ In aggregative games, conjectures can be seen as beliefs about the reaction of the aggregate. Such beliefs determine players' strategies via a (conjectural variations) equilibrium, in which each player's strategy is a best response given the player's conjecture about the reaction of the aggregate to a deviation.

Generally, players with different conjectures will play different strategies in equilibrium and thus get different payoffs. If there is an evolutionary process selecting players on the basis of these payoffs, then some conjectures will perform better than others. Suppose that in a large (infinite) population agents are randomly matched to play a given finite-player aggregative game. This paper shows that in well-behaved games the evolutionarily stable constant conjectures must be consistent at equilibrium: the beliefs about the reaction to a (small) deviation from equilibrium coincide with the marginal change in the aggregate, derived from the players' best response functions. On the other hand, evolutionary stability for finite populations (where all players interact in the same game) selects zero conjectural variations: players believe that the aggregate does not change if their strategy changes. Such behavior is akin to the price-taking behavior in the standard perfect competition model (as noted e.g. in Kamien and Schwartz, 1983).

Players with consistent conjecture correctly anticipate the reaction of the aggregate. Nevertheless, it is not obvious why they should get a higher payoff in evolutionary terms, since other players may adjust their behavior to such a conjecture. A consistent conjecture, however, does not have a detrimental strategic effect; indeed, consistency takes this strategic effect into account. In a finite population, relative payoffs are important in evolutionary terms. In symmetric games, the effect of the aggregate is the same on any player and thus

\footnotetext{
${ }^{1}$ Conjectural variations are also used in empirical industrial organization (see e.g. Belleflamme and Peitz, 2010, pp. 70-71, and references there) and in policy analysis (see a report for the former main competition authority in the UK, Office of Fair Trading, 2011).
} 
cancels out from the relative payoff evaluation. Players with zero conjectures behave as if the aggregate does not change thus mimicking the condition for maximizing relative payoffs.

The results generalize and combine several previous observations in the literature about evolutionary justifications of conjectures. For the infinite population case, Dixon and Somma (2003) and Müller and Normann (2005) showed that consistent conjectures have evolutionary foundations in certain duopoly games, while Possajennikov (2009) generalized the result to arbitrary well-behaved two-player games. Reddy Rachapalli and Kulshreshtha (2013) obtained the same result in a linear-quadratic oligopoly model. The present paper extends the result to arbitrary well-behaved $n$-player aggregative games. For the finite population case Possajennikov (2003) and Alós-Ferrer and Ania (2005) provided evolutionary background for aggregate-taking behavior in aggregative games. The present paper shows that this result can be reinterpreted via conjectures, since aggregate-taking behavior is equivalent to zero conjectural variation about the change in the aggregate. ${ }^{2}$

\section{Aggregative Games and Conjectures}

A symmetric aggregative game on the real line is given by $G=(N, X, u)$, where $N=$ $\{1, \ldots, n\}$ is the set of players, $X \subset \mathbb{R}$ is the strategy set, common across players, and $u\left(x_{i}, A\right)=u_{i}\left(x_{i}, A\right): X \times \mathbb{R} \rightarrow \mathbb{R}$ is the payoff function for each player $i$. Here, $A=$ $g\left(x_{1}, \ldots, x_{n}\right): X^{n} \rightarrow \mathbb{R}$ is the aggregate of players' strategies, such that for any permutation $\pi$ on the set of players $g\left(\pi\left(x_{1}\right), \ldots, \pi\left(x_{n}\right)\right)=g\left(x_{1}, \ldots, x_{n}\right)$. The game is assumed to be well behaved: the set $X$ is convex and the functions $u$ and $g$ are twice continuously differentiable.

A player $i$ 's conjecture $r_{i}=\left(d A / d x_{i}\right)^{e} \in R$, where $R$ is a convex subset of the real line $\mathbb{R}$, is a number representing the player's belief, or expectation, about the change in the aggregate in response to a marginal change in the player's own strategy. It is assumed that players entertain constant conjectures: $r_{i}$ does not vary with $x_{1}, \ldots, x_{n}$.

Given the conjecture $r_{i}$, player $i$ maximizes the payoff $u\left(x_{i}, A\right)$. If the solution of the player's maximization problem is interior, then the first-order condition $\partial u / \partial x_{i}\left(x_{i}, A\right)+$ $\partial u / \partial A\left(x_{i}, A\right) \cdot\left(d A / d x_{i}\right)^{e}=0$ holds. Thus

$$
F_{i}\left(x_{i}, A ; r_{i}\right):=\frac{\partial u}{\partial x_{i}}\left(x_{i}, A\right)+\frac{\partial u}{\partial A}\left(x_{i}, A\right) \cdot r_{i}=0 .
$$

For each player $i$, it is assumed that the optimal choice is determined by this equation.

Suppose that all $n$ players have some conjectures. If each player's solution of the payoff maximization problem is interior, then a (conjectural variations) equilibrium is characterized

\footnotetext{
${ }^{2}$ Müller and Normann (2007) showed that in a duopoly, finite population evolutionary stability indeed leads to a result that would be obtained with zero conjectures about the aggregate.
} 
by the following equations:

$$
\begin{aligned}
F_{1}\left(x_{1}, A ; r_{1}\right) & =0 \\
\cdots & \\
F_{n}\left(x_{n}, A ; r_{n}\right) & =0 \\
A-g\left(x_{1}, \ldots, x_{n}\right) & =0 .
\end{aligned}
$$

Definition 1 Strategy vector $\mathbf{x}^{*}=\left(x_{1}^{*}(\mathbf{r}), \ldots, x_{n}^{*}(\mathbf{r})\right)$, together with the value of the aggregate $A^{*}=A^{*}(\mathbf{r})=g\left(x_{1}^{*}(\mathbf{r}), \ldots, x_{n}^{*}(\mathbf{r})\right)$, is a conjectural variation equilibrium (CVE) for a conjecture vector $\mathbf{r}=\left(r_{1}, \ldots, r_{n}\right)$ if $\mathbf{x}^{*}, A^{*}$ satisfy the system of equations (2) for this $\mathbf{r}$.

In general a CVE may not exist or there may be multiple equilibria. An equilibrium selection assigns for each vector $\mathbf{r}$ a unique equilibrium. It is assumed in the sequel that a locally well behaved (i.e. differentiable) equilibrium selection $\mathbf{x}^{*}(\mathbf{r}), A^{*}(\mathbf{r})$ exists for the relevant values of conjectures. In a symmetric game, if conjectures are symmetric $\left(r_{i}=r_{j}\right.$ for all $i, j)$, then, whenever a CVE exists, there exists a symmetric CVE with $x_{i}^{*}(\mathbf{r})=x_{j}^{*}(\mathbf{r})$ for all $i, j$. The equilibrium selection is assumed to select this symmetric CVE.

Certain values of conjectures represent well-known solution concepts. In a standard Nash equilibrium analysis, the actions of the other players are fixed, thus $r_{i}=\left(d A / d x_{i}\right)^{e}=$ $\partial g / \partial x_{i}$. The current setting allows for many more conjectures, some of which turn out to be relevant in terms of evolution. For example, a player with zero conjectural variation $r_{i}=\left(d A / d x_{i}\right)^{e}=0$ believes that a change in his or her own action does not affect the aggregate (aggregate-taking behavior, Possajennikov, 2003, and Alós-Ferrer and Ania, 2005).

To define a (symmetric) consistent conjecture, suppose that players' conjectures are $r_{i}=r_{j}$ for all $i, j$ and imagine that player $i$ 's strategy $x_{i}$ is fixed (the definition is based on the definition for the Cournot oligopoly context in Perry, 1982, which generalized the definitions for duopoly in Laitner, 1980, and Bresnahan, 1981). The remaining $n-1$ players play a CVE of the reduced game with player $i$ 's strategy fixed at $x_{i}$. Then there are $n$ equations characterizing the CVE of the reduced game:

$$
\begin{aligned}
F_{j}\left(x_{j}, A ; r_{j}\right) & =0, j \neq i \\
A-g\left(x_{1}, \ldots, x_{n}\right) & =0 .
\end{aligned}
$$

For various $x_{i}$, this system implicitly defines reaction functions of the other players $x_{j}^{* *}\left(x_{i}\right)$ and $A^{* *}\left(x_{i}\right)=g\left(x_{1}^{* *}\left(x_{i}\right), \ldots, x_{i}, \ldots, x_{n}^{* *}\left(x_{i}\right)\right)$. It is assumed that the selection of the reaction functions is such that for a symmetric conjecture vector $\mathbf{r}$ and a corresponding symmetric $\mathrm{CVE} \mathrm{x}^{*}, A^{*}$, if $x_{i}=x_{i}^{*}$, then $x_{j}^{* *}\left(x_{i}^{*}\right)=x_{j}^{*}$ for all $j \neq i$ and $A^{* *}\left(x_{i}^{*}\right)=A^{*}$, since $x_{j}^{*}, A^{*}$ are then a solution of system (3). For a symmetric $\mathbf{r}$ with $r_{i}=r^{C}$ for all $i$ define 
Definition 2 Conjecture $r^{C}$ is consistent if for any $i$

$$
r^{C}=\frac{d A^{* *}}{d x_{i}}\left(x_{i}^{*}\right)=\frac{d g}{d x_{i}}\left(x_{1}^{* *}\left(x_{i}^{*}\right), \ldots, x_{i}^{*}, \ldots, x_{n}^{* *}\left(x_{i}^{*}\right)\right),
$$

where $x_{j}^{* *}\left(x_{i}\right), A^{* *}\left(x_{i}\right)$ is a differentiable selection of reaction functions with $x_{j}^{* *}\left(x_{i}^{*}\right)=x_{j}^{*}$ for $j \neq i, A^{* *}\left(x_{i}^{*}\right)=A^{*}$, where $\left(x_{1}^{*}, \ldots, x_{n}^{*}\right), A^{*}$ is a symmetric CVE for $\mathbf{r}$.

The definition means that a conjecture is consistent if the belief about the change in the aggregate is equal to the actual marginal change that would arise from the (reduced game) reactions of the other players to a deviation by one player from the CVE profile $\mathbf{x}^{*}$.

The consistency concept addresses some of the shortcomings inherent in arbitrary conjectures, but it does so only partially. It is a static concept, not looking at further reactions to the first reaction to a deviation, and it can be argued that even this first reaction is not properly modeled (see e.g. Makowski, 1987). However, as was shown for two-player games (Dixon and Somma, 2003, and Müller and Normann, 2005, for specific symmetric duopoly contexts and Possajennikov, 2009, more generally) and in a linear-quadratic oligopoly context (Reddy Rachapalli and Kulshreshtha, 2013), it turns out that consistent conjectures have an evolutionary foundation also in the general context of $n$-player aggregative games.

\section{Evolutionary Stability of Conjectures}

\subsection{Evolutionary stability in an infinite population}

Imagine that players are endowed with some conjectures. The previous section characterized players' (interior) CVE strategies. Substituting the equilibrium strategies into the payoff function, the payoff of player $i$ is $u\left(x_{i}^{*}(\mathbf{r}), A^{*}(\mathbf{r})\right)$.

Consider the following interpretation of the choice of conjecture for a player. There is a large (infinite) population of agents that are randomly matched to play the game $G$. Each of the agents is endowed with a conjecture and they play a CVE. Agents with different conjectures will get different payoffs in this CVE. An evolutionary interpretation is that the agents that get a higher payoff are more likely to survive or to reproduce. It is assumed here that conjectures (player's beliefs) evolve much slower than players' choices of strategies: belief is a player's innate characteristic that changes only slowly, while the player's strategy can be quickly adapted to the opponents' choices. $^{3}$

More precisely, consider a population of agents, all with the same conjecture $r^{E S}$. Suppose that some agents with a different conjecture $r^{\prime} \neq r^{E S}$ appear. In a random matching

\footnotetext{
${ }^{3}$ A good overview of this 'indirect evolution approach' can be found in Heifetz et al. (2007); see references there for applications of the approach.
} 
process, an agent can end up playing the game against $k$ players with conjecture $r^{E S}$ and $n-k$ players with conjecture $r^{\prime}$, with higher $k$ more likely than lower $k$ if the proportion of players with conjecture $r^{\prime}$ is small. Adapting the definition of evolutionary stability originated in Maynard Smith and Price (1973), let $x_{i}^{*}(r ; k), A^{*}(r ; k)$ denote the equilibrium selection if player $i$ has conjecture $r, k$ players have conjecture $r^{E S}$ and $n-k-1$ players have conjecture $r^{\prime}$. Then for a symmetric conjecture vector $\mathbf{r}$ with $r_{i}=r^{E S}$ for all $i$

Definition 3 Conjecture $r^{E S}$ is evolutionarily stable if

$$
u\left(x_{i}^{*}\left(r^{E S} ; n-1\right), A^{*}\left(r^{E S} ; n-1\right)\right) \geq u\left(x_{i}^{*}\left(r^{\prime} ; n-1\right), A^{*}\left(r^{\prime} ; n-1\right)\right)
$$

for any $r^{\prime} \neq r^{E S}$, and there exists $k^{*} \in\{0, \ldots, n-1\}$ such that if for all $k \in\left\{k^{*}+1, \ldots, n-1\right\}$ it holds that $u\left(x_{i}^{*}\left(r^{E S} ; k\right), A^{*}\left(r^{E S} ; k\right)\right)=u\left(x_{i}^{*}\left(r^{\prime} ; k\right), A^{*}\left(r^{\prime} ; k\right)\right)$ then

$$
u\left(x_{i}^{*}\left(r^{E S} ; k^{*}\right), A^{*}\left(r^{E S} ; k^{*}\right)\right)>u\left(x_{i}^{*}\left(r^{\prime} ; k^{*}\right), A^{*}\left(r^{\prime} ; k^{*}\right)\right),
$$

where $x^{*}(\mathbf{r}), A^{*}(\mathbf{r})$ is a differentiable CVE selection.

The first condition of the definition considers the most likely situation of being matched with $n-1$ agents having conjecture $r^{E S}$, and requires an agent with the conjecture $r^{E S}$ to get a (weakly) better payoff than an agent with another conjecture. If payoffs of the two conjectures are the same in this situation, then the second condition requires an agent with conjecture $r^{E S}$ to have a higher payoff in the most likely situation in which the conjectures lead to different payoffs. The conditions ensure that an agent with conjecture $r^{E S}$ has a higher expected payoff than an agent with conjecture $r^{\prime} \neq r^{E S}$ under random matching in an infinite population if the proportion of agents with conjecture $r^{\prime}$ is arbitrarily small.

From condition (4) of the definition, $r^{E S}$ maximizes $u\left(x_{i}^{*}\left(r_{i} ; n-1\right), A^{*}\left(r_{i} ; n-1\right)\right)$ as a function of $r_{i}$. A necessary condition for an interior maximum is $d u / d r_{i}=0$ at $r^{E S}$, or ${ }^{4}$

$$
\frac{\partial u}{\partial x_{i}} \frac{\partial x_{i}^{*}}{\partial r_{i}}+\frac{\partial u}{\partial A} \frac{\partial A^{*}}{\partial r_{i}}=0
$$

If $\partial u / \partial A \neq 0$ and $\partial x_{i}^{*} / \partial r_{i} \neq 0$, the left hand side can be rewritten as

$$
\frac{\partial u / \partial x_{i}}{\partial u / \partial A}+\frac{\partial A^{*} / \partial r_{i}}{\partial x_{i}^{*} / \partial r_{i}}=0
$$

From equation $(1),-\left(\partial u / \partial x_{i}\right) /(\partial u / \partial A)=r_{i}$ at the solution of player $i$ 's maximization problem. Thus if $r^{E S}$ is an evolutionarily stable conjecture, then

$$
r^{E S}=\frac{\partial A^{*} / \partial r_{i}}{\partial x_{i}^{*} / \partial r_{i}}
$$

\footnotetext{
${ }^{4}$ In the sequel, the arguments of the derivatives are omitted to save space. It is understood that the appropriate derivatives are evaluated at $\mathbf{r}=\left(r^{E S}, \ldots, r^{E S}\right)$ and at $\operatorname{CVE~} \mathbf{x}^{*}(\mathbf{r}), A^{*}(\mathbf{r})$.
} 
If one treats $\partial r_{i}$ as a small change in $r_{i}$, it can be canceled from the above expression. Then $r^{E S}=\partial A^{*} / \partial x_{i}^{*}$. Recall that a conjecture is consistent if $r_{i}=d A^{* *} / d x_{i}$ at a CVE, and that at a CVE, $A^{* *}=A^{*}$. Therefore there seems to be a relationship between evolutionary stability of a conjecture and its consistency. ${ }^{5}$ Using the implicit function theorem, the sufficient conditions for this relationship are made precise in the following proposition.

Proposition 1 Suppose that at conjecture profile $\mathbf{r}$ with $r_{i}=r^{E S}$ for all $i$, where $r^{E S}$ is an evolutionarily stable conjecture, the following regularity conditions hold:

(i) There exists a solution $\mathbf{x}^{*}(\mathbf{r}), A^{*}(\mathbf{r})$ of system (2);

(ii) $\partial u / \partial A \neq 0$ at $\mathbf{x}^{*}(\mathbf{r}), A^{*}(\mathbf{r})$;

(iii) $\prod_{i=1}^{n} \frac{\partial F_{i}}{\partial x_{i}}+\sum_{i=1}^{n} \frac{\partial g}{\partial x_{i}} \frac{\partial F_{i}}{\partial A} \prod_{j \neq i} \frac{\partial F_{j}}{\partial x_{j}} \neq 0$ at $\mathbf{r}$ and $\mathbf{x}^{*}(\mathbf{r}), A^{*}(\mathbf{r})$;

(iv) $\prod_{j \neq i} \frac{\partial F_{j}}{\partial x_{j}}+\sum_{j \neq i} \frac{\partial g}{\partial x_{j}} \frac{\partial F_{j}}{\partial A} \prod_{k \neq i, k \neq j} \frac{\partial F_{k}}{\partial x_{k}} \neq 0$ at $\mathbf{r}$ and $\mathbf{x}^{*}(\mathbf{r}), A^{*}(\mathbf{r})$.

Then $r_{i}$ is a consistent conjecture.

The formal proof of the proposition is in Appendix A.1.

Thus only consistent conjectures can (generically) be evolutionarily stable. The proposition describes what happens if an interior evolutionarily stable conjecture exists but it does not guarantee that a conjecture satisfying the first-order condition (6) is evolutionarily stable. Appropriate assumptions on the concavity of functions can ensure that the firstorder condition (6) is sufficient for evolutionary stability. In such a case the converse result also holds: a consistent conjecture is evolutionarily stable. Section 4 provides an example in which the consistency of a conjecture indeed implies its evolutionary stability.

If the choice of conjectures is interpreted as a conscious choice of a player instead of the product of evolution, then the result means that only choosing a consistent conjecture can be a Nash equilibrium of the conjecture choice game (Dixon and Somma, 2003, note this result in the linear-quadratic Cournot duopoly context). With suitable modifications of the definition of consistency and evolutionary stability to allow for heterogeneous conjectures, only choosing a consistent conjecture can actually be a best response against any conjectures of the other players.

\footnotetext{
${ }^{5}$ Itaya and Dasgupta (1995) note the relationship between consistent conjectures and the ones that maximize a player's indirect payoff $u_{i}\left(x_{i}^{*}\left(r_{i}, r_{j}\right), A^{*}\left(r_{i}, r_{j}\right)\right)$ in the context of a two-player public good game, which can also be interpreted as an aggregative game.
} 


\subsection{Evolutionary stability in a finite population}

Suppose now that the population is finite of size $n$ and all agents participate in the same interaction. For this case, Schaffer (1988) introduced a finite-population evolutionary stability concept. The concept again starts with all agents having the same conjecture $r^{f E S}$ and considers the appearance of (a small proportion of) agents using a different conjecture $r^{\prime}$. Adapted to the present context of symmetric aggregative games, for a symmetric conjecture vector $\mathbf{r}$ with $r_{i}=r^{f E S}$ for all $i$

Definition 4 Conjecture $r^{f E S}$ is finite-population evolutionarily stable (fES) if,

$$
u\left(x_{i}^{*}\left(r^{f E S} ; n-2\right), A^{*}\left(r^{f E S} ; n-2\right)\right) \geq u\left(x_{i}^{*}\left(r^{\prime} ; n-1\right), A^{*}\left(r^{\prime} ; n-1\right)\right)
$$

for any $r^{\prime} \neq r^{f E S}$, and there exists $k^{*} \in\{0, \ldots, n-1\}$ such that if for all $k \in\left\{k^{*}+1, \ldots, n-\right.$ $1\}$ it holds that $u\left(x_{i}^{*}\left(r^{f E S} ; k-1\right), A^{*}\left(r^{f E S} ; k-1\right)\right)=u\left(x_{i}^{*}\left(r^{\prime} ; k\right), A^{*}\left(r^{\prime} ; k\right)\right)$ then

$$
u\left(x_{i}^{*}\left(r^{f E S} ; k^{*}-1\right), A^{*}\left(r^{f E S} ; k^{*}-1\right)\right)>u\left(x_{i}^{*}\left(r^{\prime} ; k^{*}\right), A^{*}\left(r^{\prime} ; k^{*}\right)\right),
$$

where $\mathbf{x}^{*}(\mathbf{r}), A^{*}(\mathbf{r})$ is a differentiable CVE selection.

The idea of the definition is that if one player has a conjecture $r^{\prime} \neq r^{f E S}$, then that player will get a payoff not higher than the players who keep conjecture $r^{f E S}$. In this situation, the player with conjecture $r^{\prime}$ faces $n-1$ opponents having conjecture $r^{f E S}$ while a player with conjecture $r^{f E S}$ has 1 opponent with conjecture $r^{\prime}$ and $n-2$ opponents with conjecture $r^{f E S}$. If the payoffs of the players with different conjectures are the same, then situations with $2,3, \ldots$ players with conjecture $r^{\prime}$ are considered sequentially until, for $r^{f E S}$ to be evolutionarily stable, a situation is found in which conjecture $r^{f E S}$ brings a strictly higher payoff than conjecture $r^{\prime} .^{6}$

Schaffer (1988) shows that condition (7) of the definition means that an fES strategy maximizes relative payoff $u_{i}-u_{j}$. In the current symmetric context, an fES conjecture $r^{f E S}$ can be characterized as a solution of the problem

$$
\max _{r_{1}} u_{1}\left(x_{1}^{*}\left(r_{1}, \mathbf{r}_{-1}^{f E S}\right), A^{*}\left(r_{1}, \mathbf{r}_{-1}^{f E S}\right)\right)-u_{n}\left(x_{n}^{*}\left(r_{1}, \mathbf{r}_{-1}^{f E S}\right), A^{*}\left(r_{1}, \mathbf{r}_{-1}^{f E S}\right)\right),
$$

where $\mathbf{r}_{-1}^{f E S}=\left(r^{f E S}, \ldots, r^{f E S}\right)$. The first-order condition of this maximization problem is

$$
\frac{\partial u_{1}}{\partial x_{1}} \frac{\partial x_{1}^{*}}{\partial r_{1}}+\frac{\partial u_{1}}{\partial A} \frac{\partial A^{*}}{\partial r_{1}}-\frac{\partial u_{n}}{\partial x_{n}} \frac{\partial x_{n}^{*}}{\partial r_{1}}-\frac{\partial u_{n}}{\partial A} \frac{\partial A^{*}}{\partial r_{1}}=0
$$

\footnotetext{
${ }^{6}$ Schaffer (1988)'s original definition considered various degrees of stability depending on $k^{*}$ and requiring equation (8) to hold for all $k \in\left\{k^{*}+1, \ldots, n-1\right\}$. The present definition is closer in spirit to the one for evolutionary stability in infinite population used in the previous section.
} 
at the (interior) solution $r_{1}=r^{f E S}$. At a symmetric equilibrium with $x_{1}^{*}=x_{n}^{*}$ corresponding to the symmetric conjecture vector $\mathbf{r}^{f E S}, \partial u_{1} / \partial x_{1}=\partial u_{n} / \partial x_{n}$ and $\partial u_{1} / \partial A=\partial u_{n} / \partial A$. Equation (9) reduces to

$$
\frac{\partial u}{\partial x_{1}}\left(\frac{\partial x_{1}^{*}}{\partial r_{1}}-\frac{\partial x_{n}^{*}}{\partial r_{1}}\right)=0
$$

From the system of equations (2), the change in $r_{1}$ affects only the equilibrium equation (and thus the reaction function) for player 1 , thus it represents a movement along the reaction function of the other players. That $\partial x_{1}^{*} / \partial r_{1}-\partial x_{n}^{*} / \partial r_{1}=0$ means that the reaction function of the different players locally coincide (this is most clear if there are two players: $\partial x_{1}^{*} / \partial r_{1}-\partial x_{2}^{*} / \partial r_{1}=0$ means that the slope of the reaction function of player 2 at equilibrium is 1 since both $x_{1}^{*}$ and $x_{2}^{*}$ move by the same amount if $r_{1}$ changes; by symmetry the slope of player 1's reaction function is also 1). This (rather specific) case can be excluded by additional conditions. ${ }^{7}$ Then $\partial u / \partial x_{1}=0$ and equation (1) imply that $r^{f E S}=0$.

Proposition 2 Suppose that at conjecture profile $\mathbf{r}$ with $r_{i}=r^{f E S}$ for all $i$, where $r^{f E S}$ is a finite-population evolutionarily stable conjecture, the regularity conditions (i)-(iii) of Proposition 1 hold and the following additional conditions are satisfied:

(v) $\frac{\partial F_{i}}{\partial x_{i}} \neq 0$ at $\mathbf{r}$ and $\mathbf{x}^{*}(\mathbf{r}), A^{*}(\mathbf{r})$;

(vi) $\frac{\partial F_{i}}{\partial x_{i}}+n \frac{\partial g}{\partial x_{i}} \frac{\partial F_{i}}{\partial A} \neq 0$ at $\mathbf{r}$ and $\mathbf{x}^{*}(\mathbf{r}), A^{*}(\mathbf{r})$.

Then $r^{f E S}=0$.

The proof of the proposition is in Appendix A.2.

The result is related to the result in Possajennikov (2003), where the coincidence of the first-order conditions for aggregate-taking and finite-population evolutionarily stable behaviors is shown. In the current setting, a zero conjectural variation $r_{i}=0$ means aggregate-taking behavior: player $i$ believes that the aggregate does not change if the player changes his or her strategy. That the finite-population evolutionarily stable conjecture $r^{f E S}=0$ is then another manifestation of the connection between aggregate-taking and finite-population evolutionarily stable behaviors.

\section{Example: linear-quadratic aggregative games}

Consider the following game with $n$ players. Player $i$ chooses $x_{i} \in X \subset \mathbb{R}_{+}$. Suppose that the payoff function is

$$
u\left(x_{i}, A\right)=a x_{i}-\frac{c}{2} x_{i}^{2}+b x_{i} A,
$$

\footnotetext{
${ }^{7}$ The conditions exclude, for example, the game of 'matching the average' with payoff function $u_{i}\left(x_{i}, A\right)=$ $-\left(x_{i}-A / n\right)^{2}$ with $A=x_{1}+\ldots+x_{n}$, in which any symmetric profile $\mathbf{x}^{*}$ is a CVE for many conjectures.
} 
where $A=x_{1}+\ldots+x_{n}$ is the aggregate, $a, c>0$ and $b \neq 0$. Proposition 1 shows that only consistent conjectures can be evolutionarily stable in an infinite population. Hence the search for evolutionarily stable conjectures can be restricted to consistent ones.

Suppose that each player has some conjecture $r_{i}=\left(d A / d x_{i}\right)^{e}$. The necessary conditions for profit maximization at an interior solution for each player are $a-c x_{i}+b r_{i} x_{i}+b A=0$ for $i=1, \ldots, n$. These equations characterize a CVE if the second-order conditions $-c+2 b r_{i}<$ 0 are satisfied, which holds for $r_{i}<c /(2 b)$ if $b>0$ and for $r_{i}>c /(2 b)$ if $b<0$.

To find consistent conjectures, suppose that the players other than player $i$ all have the same conjecture $r^{C}$. The necessary conditions for profit maximization for each of these $n-1$ players are $a-c x_{j}+b^{C} x_{j}+b A=0$. Adding up these first-order conditions gives $(n-1) a-c\left(A-x_{i}\right)+b r^{C}\left(A-x_{i}\right)+(n-1) b A=0$. Therefore

$$
A^{* *}=\frac{c-b r^{C}}{c-b r^{C}-(n-1) b} x_{i}+\frac{(n-1) a}{c-b r^{C}-(n-1) b} .
$$

A symmetric consistent conjecture is then characterized by

$$
r^{C}=\frac{d A^{* *}}{d x_{i}}=\frac{c-b r^{C}}{c-b r^{C}-(n-1) b} .
$$

If $b>0$, this quadratic equation can have zero, one, or two solutions for $r^{C}$ satisfying the second-order condition $r^{C}<c /(2 b)$. The case $b<0$ is simpler. If $b<0$, equation (11) has one root between 0 and 1 , thus satisfying the second-order condition $r^{C}>c /(2 b)$. For the other root, $r^{C}<c / b<c /(2 b)$, violating the second-order condition. Thus for any $n>1$ there is a unique symmetric consistent $r^{C} \in(0,1)$.

The case $b<0$ presents a typical economic example of an aggregative game, the linearquadratic Cournot oligopoly. Players are firms, they choose production quantities $q_{i}$, the total production is $Q=q_{1}+\ldots+q_{n}$, the inverse demand function is $P(Q)=a+b Q$ and the cost function is $C\left(q_{i}\right)=\frac{c}{2} q_{i}^{2}$. For this setting, Dixon and Somma (2003) and Müller and Normann (2005) showed that consistent conjectures are the only evolutionarily stable ones for $n=2$ and Reddy Rachapalli and Kulshreshtha (2013) extended the result to the $n$-player case. The results in the present paper allow to simplify the analysis by focusing on consistent conjectures as the unique candidate for evolutionarily stable conjectures. The results are also valid for demand-cost specifications other than linear-quadratic and, in addition, allow considering finite-population evolutionary stability of conjectures.

For a finite population, Proposition 2 shows that only the conjecture $r=0$ can be evolutionarily stable. Consider the relative payoff maximization problem

$$
\max _{r_{1}}\left(a+b A^{*}\left(r_{1}, \mathbf{r}_{-1}\right)\right)\left(x_{1}^{*}\left(r_{1}, \mathbf{r}_{-1}\right)-x_{n}^{*}\left(r_{1}, \mathbf{r}_{-1}\right)\right)-\frac{c}{2}\left(\left(x_{1}^{*}\left(r_{1}, \mathbf{r}_{-1}\right)\right)^{2}-\left(x_{n}^{*}\left(r_{1}, \mathbf{r}_{-1}\right)\right)^{2}\right)
$$


where the conjectures of $n-1$ players are $\mathbf{r}_{-1}=(0, \ldots, 0)$.

The equilibrium values of $A^{*}, x_{1}^{*}$ and $x_{i}^{*}$ are found from the conditions $a+b A^{*}-(c-$ $\left.b r_{1}\right) x_{1}^{*}=0$ and $n-1$ conditions $a+b A^{*}-c x_{i}^{*}=0$ for $i \neq 1$. The last conditions add up to $(n-1) a-(c-(n-1) b) A^{*}+c x_{1}^{*}=0$. Then

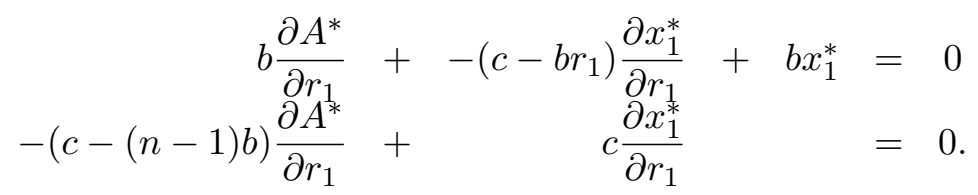

Let $D=b c-\left(c-b r_{1}\right)(c-(n-1) b)$. Since $r_{1}>c /(2 b)>c / b, D<0$. Then $\partial A^{*} / \partial r_{1}=$ $(1 / D)\left(-b x_{1}^{*}\right) c<0$ and $\partial x_{1}^{*} / \partial r_{1}=(1 / D)\left(-b x_{1}^{*}\right)(c-(n-1) b)<0$ at an interior $x_{1}^{*}$. Note also that since $x_{n}^{*}=(1 /(n-1))\left(A^{*}-x_{1}^{*}\right), \partial x_{n}^{*} / \partial r_{1}=(1 / D)\left(-b^{2} x_{1}^{*}\right)>0$.

The first order condition for maximizing function (12) is

$$
\left(a+b A^{*}\right)\left(\frac{\partial x_{1}^{*}}{\partial r_{1}}-\frac{\partial x_{n}^{*}}{\partial r_{1}}\right)+b \frac{\partial A^{*}}{\partial r_{1}}\left(x_{1}^{*}-x_{n}^{*}\right)-c x_{1}^{*} \frac{\partial x_{1}^{*}}{\partial r_{1}}+c x_{n}^{*} \frac{\partial x_{n}^{*}}{\partial r_{1}}=0 .
$$

From the equilibrium equations, $a+b A^{*}-c x_{1}^{*}=-b r_{1} x_{1}^{*}$ and $a+b A^{*}-c x_{n}^{*}=0$ thus the condition becomes $b\left(\partial A^{*} / \partial r_{1}\right)\left(x_{1}^{*}-x_{n}^{*}\right)-b x_{1}^{*} r_{1}\left(\partial x_{1}^{*} / \partial r_{1}\right)=0$. If $r_{1}>0$, then $x_{1}^{*}<x_{n}^{*}$ and thus both terms on the left-hand side are negative. Similarly, if $r_{1}<0$, then $x_{1}^{*}>x_{n}^{*}$ and thus both terms are positive. Thus the only solution of the first order condition is $r^{f E S}=0$, and the signs of the terms imply that the function is strictly maximized at $r^{f E S}=0$.

Summarizing the results,

Proposition 3 In the linear-quadratic game with $b<0$ (including Cournot oligopoly),

(i) the consistent conjecture $r^{C} \in(0,1)$ satisfying $r^{C}=\frac{c-b r^{C}}{c-b r^{C}-(n-1) b}$ is the unique evolutionarily stable conjecture for an infinite population;

(ii) the aggregate-taking conjecture $r=0$ is the unique finite-population evolutionarily stable conjecture.

Note that zero conjectures about the aggregate, which characterize aggregate-taking behavior, mean competitive (Walrasian) equilibrium for any number $n$ of firms in this setting.

\section{Conclusion}

Evolutionary stability means that a player with a different conjecture would get a lower payoff than a player with the currently prevailing conjecture. If players in an infinite population are randomly matched in groups of $n$ to play a given game, only consistent conjecture can be evolutionarily stable. A consistent conjecture correctly anticipates the 
strategic reaction of other players and thus leads to the best payoff. This intuition should work more generally; however, with many players and many conjectures the dimensionality of the analysis increases considerably. Concentrating on aggregative games allows precise statements regarding the relationship between consistency and evolutionary stability.

Focusing on aggregative games also allows linking evolutionary stability in finite populations with a particular conjecture $r=0$ representing aggregate-taking behavior. A zero conjecture in symmetric aggregative games commits a player to a behavior that maximizes the difference between the player's payoffs and the payoff of another player. If evolution selects players on the basis of relative payoffs, as it does in a finite population, such a behavior (and therefore the associated zero conjecture) can be evolutionarily stable.

The results are illustrated on the example of aggregative game that includes linearquadratic Cournot oligopoly. In such a game (with $b<0$ ) the converse results also hold: the consistent conjecture is evolutionarily stable and the zero conjecture leading to the aggregate-taking behavior (which means price-taking behavior in the oligopoly context) is finite-population evolutionarily stable.

\section{A Proofs}

\section{A.1 Proof of Proposition 1}

To simplify notation and because of symmetry, consider $i=1$. From the system of equations (2), at its solution $x^{*}(\mathbf{r}), A^{*}(\mathbf{r})$,

$$
\begin{aligned}
& \frac{\partial F_{1}}{\partial x_{1}} \frac{\partial x_{1}^{*}}{\partial r_{1}}+0+\ldots+0+\frac{\partial F_{1}}{\partial A} \frac{\partial A^{*}}{\partial r_{1}}=-\frac{\partial F_{1}}{\partial r_{1}} \\
& 0+\frac{\partial F_{2}}{\partial x_{2}} \frac{\partial x_{2}^{*}}{\partial r_{1}}+\ldots+\ldots+\frac{\partial F_{2}}{\partial A} \frac{\partial A^{*}}{\partial r_{1}}=0 \\
& 0+0+\ldots+\frac{\partial F_{n}}{\partial x_{n}} \frac{\partial x_{n}^{*}}{\partial r_{1}}+\frac{\partial F_{n}}{\partial A} \frac{\partial A^{*}}{\partial r_{1}}=0 \\
& -\frac{\partial g}{\partial x_{1}} \frac{\partial x_{1}^{*}}{\partial r_{1}}-\frac{\partial g}{\partial x_{2}} \frac{\partial x_{2}^{*}}{\partial r_{1}}-\ldots-\frac{\partial g}{\partial x_{n}} \frac{\partial x_{n}^{*}}{\partial r_{1}}+\frac{\partial A^{*}}{\partial r_{1}}=0 .
\end{aligned}
$$

Let

$$
D=\left(\begin{array}{ccccc}
\frac{\partial F_{1}}{\partial x_{1}} & 0 & \ldots & 0 & \frac{\partial F_{1}}{\partial A} \\
0 & \frac{\partial F_{2}}{\partial x_{2}} & \cdots & 0 & \frac{\partial F_{2}}{\partial A} \\
\vdots & \cdots & \ddots & \ldots & \vdots \\
0 & 0 & \cdots & \frac{\partial F_{n}}{\partial x_{n}} & \frac{\partial F_{n}}{\partial A} \\
-\frac{\partial g}{\partial x_{1}} & -\frac{\partial g}{\partial x_{2}} & \cdots & -\frac{\partial g}{\partial x_{n}} & 1
\end{array}\right) .
$$


Then

$$
|D|=\prod_{i=1}^{n} \frac{\partial F_{i}}{\partial x_{i}}+\sum_{i=1}^{n} \frac{\partial g}{\partial x_{i}} \frac{\partial F_{i}}{\partial A} \prod_{j \neq i} \frac{\partial F_{j}}{\partial x_{j}} .
$$

Condition (iii) of the proposition means that $|D| \neq 0$. By Cramer's rule,

$$
\frac{\partial x_{1}^{*}}{\partial r_{1}}=\frac{1}{|D|}\left|\begin{array}{ccccc}
-\frac{\partial F_{1}}{\partial r_{1}} & 0 & \ldots & 0 & \frac{\partial F_{1}}{\partial A} \\
0 & \frac{\partial F_{2}}{\partial x_{2}} & \ldots & 0 & \frac{\partial F_{2}}{\partial A} \\
\vdots & \ldots & \ddots & \ldots & \vdots \\
0 & 0 & \ldots & \frac{\partial F_{n}}{\partial x_{n}} & \frac{\partial F_{n}}{\partial A} \\
0 & -\frac{\partial g}{\partial x_{2}} & \ldots & -\frac{\partial g}{\partial x_{n}} & 1
\end{array}\right|=\frac{1}{|D|}\left(-\frac{\partial F_{1}}{\partial r_{1}}\right)\left|D_{-1}\right|
$$

where

$$
D_{-1}=\left(\begin{array}{cccc}
\frac{\partial F_{2}}{\partial x_{2}} & \ldots & 0 & \frac{\partial F_{2}}{\partial A} \\
\ldots & \ddots & \ldots & \vdots \\
0 & \ldots & \frac{\partial F_{n}}{\partial x_{n}} & \frac{\partial F_{n}}{\partial A} \\
-\frac{\partial g}{\partial x_{2}} & \cdots & -\frac{\partial g}{\partial x_{n}} & 1
\end{array}\right)
$$

and thus

$$
\left|D_{-1}\right|=\prod_{i=2}^{n} \frac{\partial F_{i}}{\partial x_{i}}+\sum_{i=2}^{n} \frac{\partial g}{\partial x_{i}} \frac{\partial F_{i}}{\partial A} \prod_{j \neq i, j \neq 1} \frac{\partial F_{j}}{\partial x_{j}}
$$

Also,

$$
\frac{\partial A^{*}}{\partial r_{1}}=\frac{1}{|D|}\left|\begin{array}{ccccc}
\frac{\partial F_{1}}{\partial x_{1}} & 0 & \ldots & 0 & -\frac{\partial F_{1}}{\partial r_{1}} \\
0 & \frac{\partial F_{2}}{\partial x_{2}} & \cdots & 0 & 0 \\
\vdots & \ddots & \ddots & \ddots & \vdots \\
0 & 0 & \ldots & \frac{\partial F_{n}}{\partial x_{n}} & 0 \\
-\frac{\partial g}{\partial x_{1}} & -\frac{\partial g}{\partial x_{2}} & \cdots & -\frac{\partial g}{\partial x_{n}} & 0
\end{array}\right|=-\frac{1}{|D|} \frac{\partial F_{1}}{\partial r_{1}} \frac{\partial g}{\partial x_{1}} \prod_{i \neq 1} \frac{\partial F_{i}}{\partial x_{i}}
$$

Therefore,

$$
\frac{\partial A^{*} / \partial r_{1}}{\partial x_{1}^{*} / \partial r_{1}}=\frac{-\frac{\partial F_{1}}{\partial r_{1}} \frac{\partial f}{\partial x_{1}} \prod_{i \neq 1} \frac{\partial F_{i}}{\partial x_{i}}}{\left(-\frac{\partial F_{1}}{\partial r_{1}}\right)\left(\prod_{i=2}^{n} \frac{\partial F_{i}}{\partial x_{i}}+\sum_{i=2}^{n} \frac{\partial g}{\partial x_{i}} \frac{\partial F_{i}}{\partial A} \prod_{j \neq i, j \neq 1} \frac{\partial F_{j}}{\partial x_{j}}\right)}=\frac{1}{\left|D_{-1}\right|} \frac{\partial g}{\partial x_{1}} \prod_{i \neq 1} \frac{\partial F_{i}}{\partial x_{i}},
$$

where condition (iv) guarantees that $\left|D_{-1}\right| \neq 0$ and condition (ii) that $\partial F_{1} / \partial r_{1} \neq 0$ (from equation (1) $\partial F_{1} / \partial r_{1}=\partial u / \partial A \neq 0$ ).

The reaction of the aggregate to independent changes in the strategy of player 1 is found 
from system (3). Using again the implicit function theorem, $d A^{* *} / d x_{1}$ can be found from

$$
\begin{aligned}
& \frac{\partial F_{2}}{\partial x_{2}} \frac{d x_{2}^{* *}}{d x_{1}}+\ldots+\quad 0+\frac{\partial F_{2}}{\partial A} \frac{d A^{* *}}{d x_{1}}=0 \\
& \begin{array}{lllll}
\cdots & \cdots & \cdots & \cdots & \cdots
\end{array} \\
& 0 \quad+\ldots+\frac{\partial F_{n}}{\partial x_{n}} \frac{d x_{n}^{* *}}{d x_{1}}+\frac{\partial F_{n}}{\partial A} \frac{d A^{* *}}{d x_{1}}=0 \\
& -\frac{\partial g}{\partial x_{2}} \frac{d x_{2}^{* *}}{d x_{1}}-\ldots-\frac{\partial g}{\partial x_{n}} \frac{d x_{n}^{* *}}{d x_{1}}+\frac{d A^{* *}}{d x_{1}}=\frac{\partial g}{\partial x_{1}} \text {. }
\end{aligned}
$$

Then

$$
\frac{d A^{* *}}{d x_{1}}=\frac{1}{\left|D_{-1}\right|}\left|\begin{array}{cccc}
\frac{\partial F_{2}}{\partial x_{2}} & \cdots & 0 & 0 \\
\vdots & \ddots & \ddots & \vdots \\
0 & \cdots & \frac{\partial F_{n}}{\partial x_{n}} & 0 \\
-\frac{\partial g}{\partial x_{2}} & \cdots & -\frac{\partial g}{\partial x_{n}} & \frac{\partial g}{\partial x_{1}}
\end{array}\right|=\frac{1}{\left|D_{-1}\right|} \frac{\partial g}{\partial x_{1}} \prod_{i \neq 1} \frac{\partial F_{i}}{\partial x_{i}}
$$

Comparing equations (13) and (14), it holds that

$$
\frac{d A^{* *}}{d x_{1}}=\frac{\partial A^{*} / \partial r_{1}}{\partial x_{1}^{*} / \partial r_{1}} .
$$

Thus if $r^{E S}$ is an evolutionarily stable conjecture, then

$$
r^{E S}=-\frac{\partial u / \partial x_{1}}{\partial u / \partial A}=\frac{\partial A^{*} / \partial r_{1}}{\partial x_{1}^{*} / \partial r_{1}}=\frac{d A^{* *}}{d x_{1}},
$$

i.e. $r^{E S}$ is consistent.

\section{A.2 Proof of Proposition 2}

Recall from the proof of Proposition 1 that

$$
\frac{\partial x_{1}^{*}}{\partial r_{1}}=\frac{1}{|D|}\left(-\frac{\partial F_{1}}{\partial r_{1}}\right)\left(\prod_{i=2}^{n} \frac{\partial F_{i}}{\partial x_{i}}+\sum_{i=2}^{n} \frac{\partial g}{\partial x_{i}} \frac{\partial F_{i}}{\partial A} \prod_{j \neq i, j \neq 1} \frac{\partial F_{j}}{\partial x_{j}}\right)
$$

in equilibrium. From system (2),

$$
\frac{\partial x_{n}^{*}}{\partial r_{1}}=\frac{1}{|D|}\left|\begin{array}{ccccc}
\frac{\partial F_{1}}{\partial x_{1}} & 0 & \ldots & -\frac{\partial F_{1}}{\partial r_{1}} & \frac{\partial F_{1}}{\partial A} \\
0 & \frac{\partial F_{2}}{\partial x_{2}} & \ldots & 0 & \frac{\partial F_{2}}{\partial A} \\
\vdots & \ddots & \ddots & \ddots & \vdots \\
0 & 0 & \ldots & 0 & \frac{\partial F_{n}}{\partial A} \\
-\frac{\partial g}{\partial x_{1}} & -\frac{\partial g}{\partial x_{2}} & \cdots & 0 & 1
\end{array}\right|=\frac{1}{|D|} \frac{\partial F_{1}}{\partial r_{1}} \frac{\partial g}{\partial x_{1}} \frac{\partial F_{n}}{\partial A} \prod_{j \neq n, j \neq 1} \frac{\partial F_{j}}{\partial x_{j}} .
$$

Simplifying the expressions above,

$$
\frac{\partial x_{1}^{*}}{\partial r_{1}}-\frac{\partial x_{n}^{*}}{\partial r_{1}}=\frac{-\partial F_{1} / \partial r_{1} \cdot\left(\partial F_{1} / \partial x_{1}\right)^{n-2}}{|D|}\left(\frac{\partial F_{1}}{\partial x_{1}}+n \frac{\partial g}{\partial x_{1}} \frac{\partial F_{1}}{\partial A}\right) .
$$


Conditions (v) and (vi) of the proposition imply that $\partial x_{1}^{*} / \partial r_{1}-\partial x_{n}^{*} / \partial r_{1} \neq 0$ (condition (ii) of Proposition 1, $\partial u / \partial A \neq 0$, implies $\left.\partial F_{i} / \partial r_{i} \neq 0\right)$.

From equation (10), if $\partial x_{1}^{*} / \partial r_{1}-\partial x_{n}^{*} / \partial r_{1} \neq 0$, then the necessary condition for finite population evolutionary stability is $\partial u / \partial x_{1}=0$. But recall that a necessary condition for player 1 to play a strategy $x_{1}^{*}$ that is a part of a CVE is given by equation (1), ( $\left.\partial u / \partial x_{1}\right)+$ $(\partial u / \partial A) r_{1}=0$. If $\partial u / \partial A \neq 0$, then the only way to satisfy the two conditions is $r_{1}=0$.

\section{Acknowledgements}

I would like to thank Alex Dickson for organizing the workshop on aggregative games in April 2011 at the University of Strathclyde, Glasgow, where the first version of the paper was presented, and Maria Montero for help in improving the presentation of the results. I also thank the associate editor and two anonymous referees for useful comments leading to more concise exposition of the results.

\section{References}

Acemoglu, D., Jensen, M.K. (2013) "Aggregate Comparative Statics", Games and Economic Behavior 81, 27-49.

Alós-Ferrer, C., Ania, A.B. (2005) "The Evolutionary Stability of Perfectly Competitive Behavior", Economic Theory 26, 497-516.

Belleflamme, P., Peitz, M. (2010) Industrial Organization: Markets and Strategies, Cambridge University Press, Cambridge.

Bresnahan, T.F. (1981) "Duopoly Models with Consistent Conjectures", American Economic Review 71, 934-945.

Corchón, L.C. (1994) "Comparative Statics for Aggregative Games. The Strong Concavity Case", Mathematical Social Sciences 28, 151-165.

Cornes, R., Hartley, R. (2012) "Fully Aggregative Games", Economics Letters 116, 631-633.

Dixon, H.D., Somma, E. (2003) "The Evolution of Consistent Conjectures", Journal of Economic Behavior \& Organization 51, 523-536.

Figuières, C., Jean-Marie, A., Quérou, N., Tidball, M. (2004) Theory of Conjectural Variations, World Scientific, London.

Heifetz, A., Shannon, C., Spiegel, Y. (2007) "What to Maximize If You Must", Journal of Economic Theory 133, 31-57. 
Itaya, J.-I., Dasgupta, D. (1995) "Dynamics, Consistent Conjectures, and Heterogeneous Agents in the Private Provision of Public Goods", Public Finance / Finances Publiques 50(3), 371-389.

Kamien, M.I., Schwartz, N.L. (1983) "Conjectural Variations", Canadian Journal of Economics 16(2), 191-211.

Laitner, J. (1980) "'Rational" Duopoly Equilibria", Quarterly Journal of Economics 95, 641-662.

Makowski, L. (1987) "Are 'Rational Conjectures' Rational?", Journal of Industrial Economics 36(1), 35-47.

Maynard Smith, J., Price, G.R. (1973) "The Logic of Animal Conflict", Nature 246 (Nov 2), 15-18.

Müller, W., Normann, H.-T. (2005) "Conjectural Variations and Evolutionary Stability: A Rationale for Consistency", Journal of Institutional and Theoretical Economics 161, 491-502.

Müller, W., Normann, H.-T. (2007) "Conjectural Variations and Evolutionary Stability in Finite Populations", Journal of Evolutionary Economics 17(1), 53-61.

Office of Fair Trading (2011) "Conjectural Variations and Competition Policy: Theory and Empirical Techniqies", report OFT1379 for the Office of Fair Trading by RBB Economics, available at http://webarchive.nationalarchives.gov.uk/20140402142426/http:// www.oft.gov.uk/shared_oft/research/CV_Competition_Policy.pdf.

Perry, M.K. (1982) "Oligopoly and Consistent Conjectural Variations", Bell Journal of Economics 13(1), 197-205.

Possajennikov, A. (2003) "Evolutionary Foundations of Aggregate-taking Behavior", Economic Theory 21, 921-928.

Possajennikov, A. (2009) "The Evolutionary Stability of Constant Consistent Conjectures", Journal of Economic Behavior \& Organization 72, 21-29.

Reddy Rachapalli, S., Kulshreshtha, P. (2013) "Evolutionarily Stable Conjectures and Social Optimality in Oligopolies", Theoretical Economics Letters 3(1), 12-18.

Schaffer, M.E. (1988) "Evolutionarily Stable Strategies for a Finite Population and a Variable Contest Size", Journal of Theoretical Biology 132, 469-478. 